\title{
Study of MPPC at Liquid Nitrogen Temperature
}

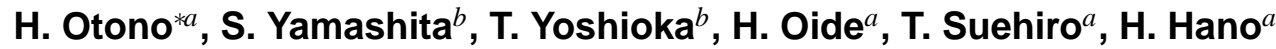 \\ ${ }^{a}$ Department of Physics, University of Tokyo \\ ${ }^{b}$ International Center for Elementary Particle Physics, University of Tokyo \\ E-mail: otono@icepp.s.u-tokyo.ac.jp
}

The Geiger-mode APD is a new semiconductor photon sensor which has high photon counting capability. Multi-Pixel Photon Counters (MPPC) is one of the Geiger-mode APD produced by Hamamatsu Photonics. In this paper, we report the basic properties such as waveform, breakdown voltage, gain ,dark noise, cross-talk, after-pulse and recovery of the MPPC at extremely low temperature, namely the ethanol with dry ice $(200 \mathrm{~K})$ and the liquid nitrogen $(77 \mathrm{~K})$.

International Workshop on new Photon-Detectors

June 27-29 2007

Kobe, Japan

${ }^{*}$ Speaker. 


\section{Introduction}

The Geiger-mode APD is a new type of photon counting device which consists of multiple APD (avalanche photo-diode) pixels operated in Geiger mode. It has many advantages compared to the PMT (insensitive to the magnetic field, low bias voltage, etc.), therefore it could be a successor of the PMT in the high energy experiment or the medical field in near future. The development started some 10 years ago in Russia [1], and the production has begun in many places in the world. The device studied in this paper is the Multi-Pixel Photon Counter (MPPC) produced by Hamamatsu Photonics (HPK) which is a type of Geiger-mode APD [2]. The MPPC has $1 \mathrm{~mm} \times 1 \mathrm{~mm}$ photosensitive surface and it consists of 1600 pixels. In the following sections, we evaluate the basic properties of 1600 pixel MPPC at $300 \mathrm{~K}$ (room temperature), 200K (ethanol with crashed dry ice) and 77K (liquid nitrogen temperature). We directly cooled the MPPC in dewar, and the cooled components are MPPC itself and the low-pass filter which is located at upstream of the MPPC. A picture of the MPPC and the setup for the measurement at low temperature are shown in Figure 1. First of all, we will show the waveforms at each temperature. We will discuss the value of the quenching resistance and the $\mathrm{p}-\mathrm{n}$ junction capacitance. Second, we will refer to the temperature dependence of the breakdown voltage. Then, we will see how the rates of dark noise and cross-talk depend on the temperature. Finally, we will discuss on the after-pulse and recovery processes at low temperature.

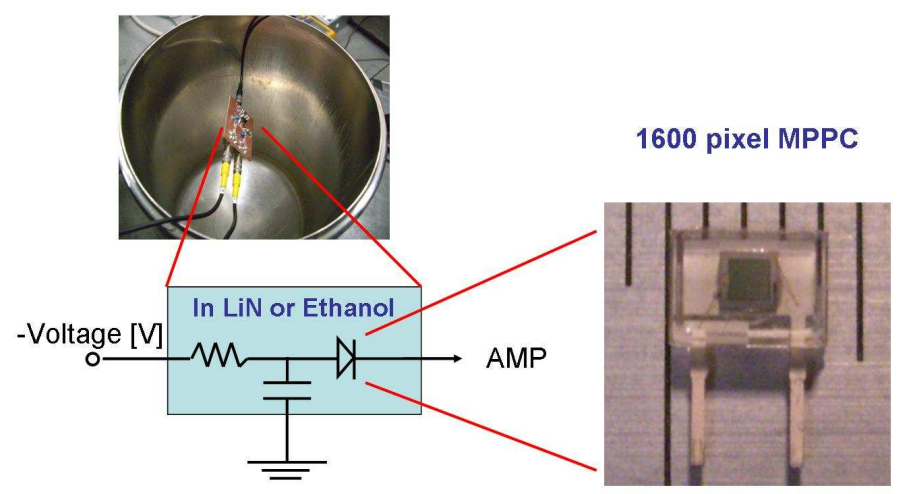

Figure 1: A schematic view of the setup for measurement at low temperature. The MPPC and the lowpass filter which is located at upstream of the MPPC are directly cooled by liquid refrigerant in the dewar. Closeup picture of the 1600 pixel MPPC is also shown in the figure.

\section{Waveform and $\mathrm{RC}$ time constant}

Figure 2 shows the waveforms observed by an oscilloscope at each temperature. We observed two components (fast and slow component) at $200 \mathrm{~K}$ and $77 \mathrm{~K}$, while we see clearly only one component at $300 \mathrm{~K}$. From the waveforms, decay constant of the slow component has been evaluated to be $5 \mathrm{nsec}, 10 \mathrm{nsec}$ and $40 \mathrm{nsec}$ for $300 \mathrm{~K}, 200 \mathrm{~K}$ and $77 \mathrm{~K}$, respectively. The slow component is considered to be related to the $R_{\text {quenching }} C_{\text {pixel }}$ time constant, where $R_{\text {quenching }}$ is the quenching resistance which is equipped pixel by pixel and $C_{\text {pixel }}$ is the p-n junction capacitance. In order to measure the $R_{\text {quenching }}$, we applied forward voltage to the MPPC and measured the current value. Figure 3 shows the measured I-V curve. The slope of the curve corresponds to the quenching 


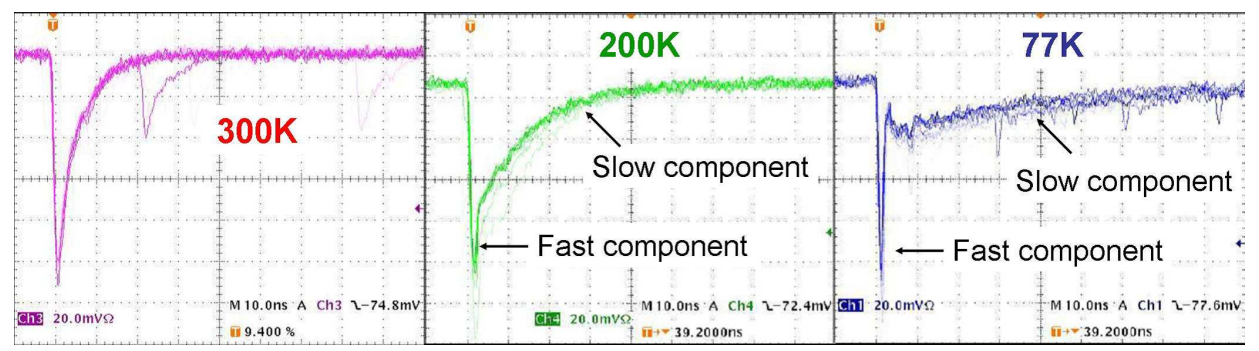

Figure 2: Waveforms observed at $300 \mathrm{~K}$ (left), 200K (middle) and $77 \mathrm{~K}$ (right). The waveforms at $200 \mathrm{~K}$ and $77 \mathrm{~K}$ seem to have two components.

resistance value when the current is sufficiently high. We use the following formula for the $C_{\text {pixel }}$ :

$$
\text { Gain }=\frac{C_{\text {pixel }}}{e}\left(V_{\text {bias }}-V_{\text {breakdown }}\right)
$$

where $e$ is elementary charge, $V_{\text {bias }}$ is bias voltage or reverse voltage and $V_{\text {breakdown }}$ is breakdown voltage. As one can see from the formula, the pixel capacitance $C_{\text {pixel }}$ is proportional to the slope of the gain curve. Figure 4 shows the measured gain curve. The breakdown voltage $V_{\text {breakdown }}$ also can be obtained from this figure. This is referred in the next section. We obtained the $R_{\text {quenching }}$ and $C_{\text {pixel }}$ values from the Figure 3 and 4 , respectively. These values are summarized in Table 1 together with the $R_{\text {quenching }} C_{\text {pixel }}$ time constant value at each temperature. By comparing with the decay constant obtained by the Figure 2 , the variation of the slow component can be explained by such effect. On the other hand, the fast component cannot be explained by the $R_{\text {quenching }} C_{\text {pixel }}$ time constant and the origin is not understood yet.

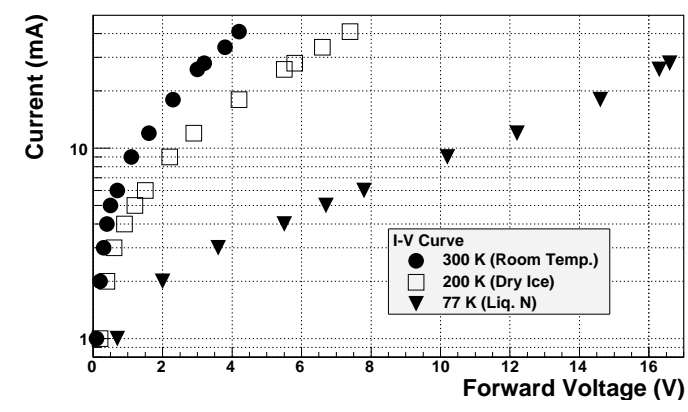

Figure 3: Current value as a function of the for- Figure 4: Gain value as a function of the bias voltward voltage for each temperature.

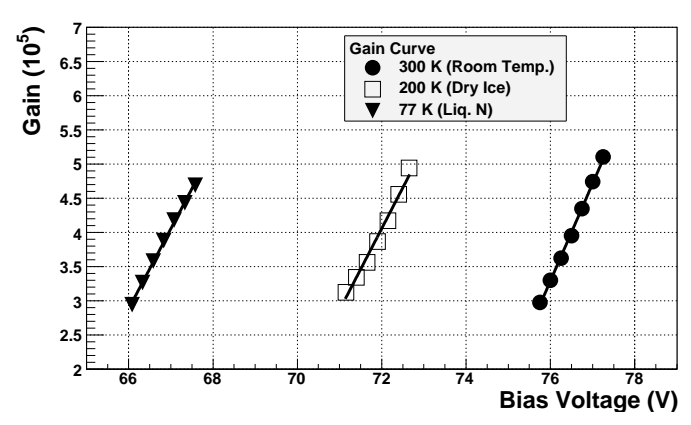

age for each temperature.

\section{Breakdown Voltage}

The Geiger-mode avalanche multiplication can be occurred above the breakdown voltage. At low temperature, the mobility of the carrier is higher than that of room temperature $\left(10^{3} \mathrm{~cm}^{2} / \mathrm{V} \cdot \mathrm{sec}\right.$ at $300 \mathrm{~K}$ and $10^{4} \mathrm{~cm}^{2} / \mathrm{V} \cdot \mathrm{sec}$ at $77 \mathrm{~K}$ [4]). Thus, as the temperature is getting lower, the breakdown voltage would be also getting lower. As already mentioned in the previous section, the breakdown 


\begin{tabular}{cccc}
\hline Temperature $(\mathrm{K})$ & $R_{\text {quenching }}(\mathrm{M} \Omega)$ & $C_{\text {pixel }}(\mathrm{fF})$ & $R_{\text {quenching }} C_{\text {pixel }}(\mathrm{nsec})$ \\
\hline 300 & 0.21 & 22.1 & 4.6 \\
200 & 0.40 & 22.0 & 8.8 \\
77 & 1.68 & 21.3 & 35.8 \\
\hline
\end{tabular}

Table 1: Measured $R_{\text {quenching }}$ and $C_{\text {pixel }}$ values at each temperature.

voltage can be obtained from eqn. 2.1 or from the Figure 4 as the intersection of the gain curve with horizontal axis. Figure 5 shows the breakdown voltage at each temperature. The temperature dependence of the breakdown voltage around room temperature has been already measured to be $50 \mathrm{mV} / \mathrm{K}$ [2]. This value is consistent with our measured value of $47 \mathrm{mV} / \mathrm{K}$.

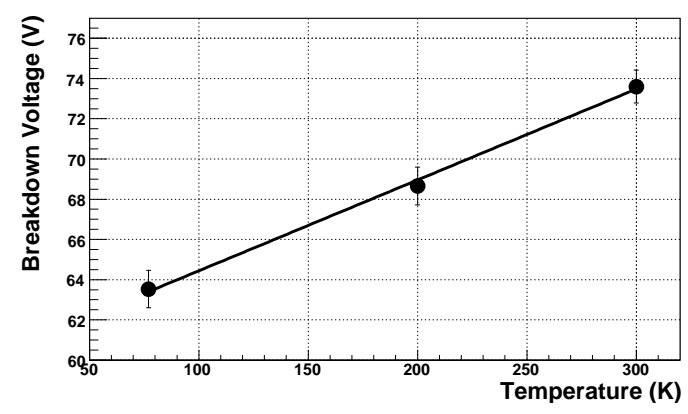

Figure 5: The breakdown voltage at each temperature. Slope of the fitted line is $47 \mathrm{mV} / \mathrm{K}$. This value is consistent with the already measured value $(50 \mathrm{mV} / \mathrm{K})$ around room temperature.

\section{Dark noise and Cross-talk}

The origin of the dark noise is thermal excited carrier or tunneling effect [1]. Typical value of the dark noise rate is about $500 \mathrm{MHz} / \mathrm{mm}^{2}$ at room temperature. The probability of thermal excitation $\left(P_{\text {thermal }}\right)$ depends on the temperature and can be expressed as $P_{\text {thermal }}(T) \propto T^{\frac{3}{2}} \times \exp \left(-\frac{E g}{2 k_{B} T}\right)$, where $E g$ is band-gap energy and $k_{B}$ is the Boltzmann constant. On the other hand, the probability of the tunneling effect doesn't depend on temperature, and it increases with the over-voltage $\left(V_{\text {bias }}-V_{\text {breakdown }}\right)$ [1, 3]. Figure 6 shows the temperature dependence of the dark noise rate. Since the measured rate at low temperature is higher than expected by thermal excitation, the tunneling effect would dominate at low temperature.

Next topic is the cross-talk. Light emission from hot carriers in silicon devices has been observed since 1950s [5]. The photon with energy higher than $1.14 \mathrm{eV}$ is emitted per $2.9 \times 10^{5}$ carriers [6]. When these photons travel to a neighboring pixel, they can trigger the next avalanche at the pixel. This phenomena is called "cross-talk". Figure 7 shows the probability that the normal pulse generates the cross-talk as a function of the over-voltage for each temperature. According to [6], the intensity and the shape of energy spectrum of the emitted photon are independent on the temperature. On the other hand, the band-gap energy is increased from $1.115 \mathrm{eV}$ at $300 \mathrm{~K}$ to $1.165 \mathrm{eV}$ at $0 \mathrm{~K}$. As a result, the cross-talk probability is expected to be slightly reduced at low temperature due to the variation of the band-gap energy. 

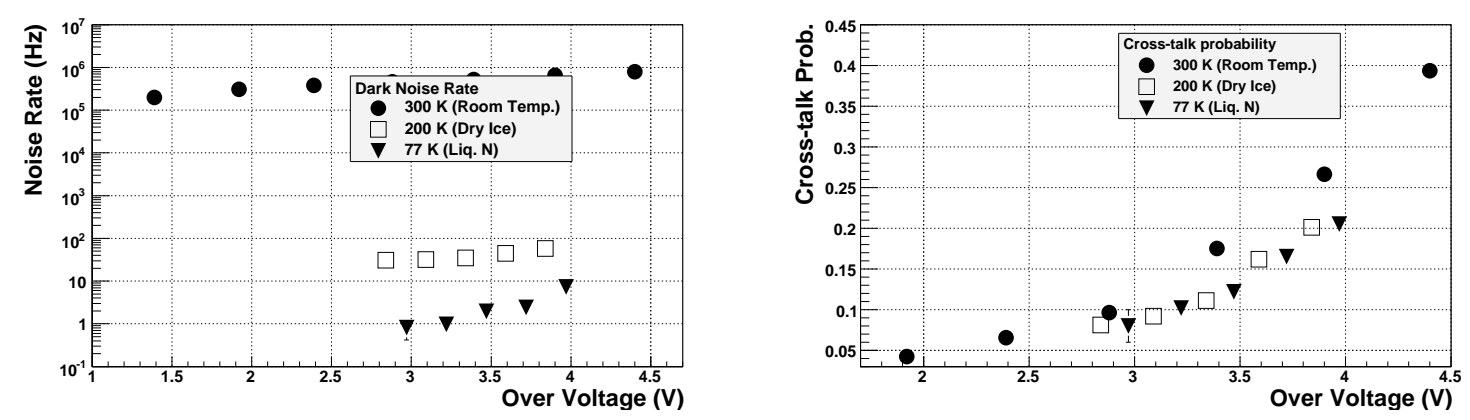

Figure 6: The dark noise consists of the thermal Figure 7: Probability that the normal pulse gennoise and the tunneling effect. The dark noise at erates the cross-talk as a function of the over$300 \mathrm{~K}$ and $77 \mathrm{~K}$ is considered to be dominated by voltage. The probability is slightly reduced at low the thermal noise and the tunneling, respectively. temperature.

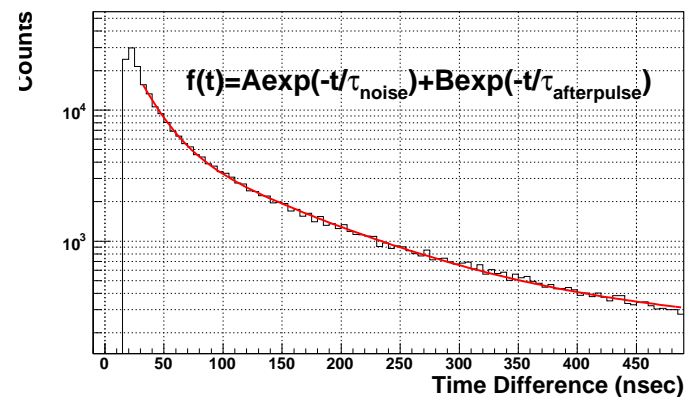

Figure 8: Distribution of the time interval between an arbitrary pulse and the next pulse.

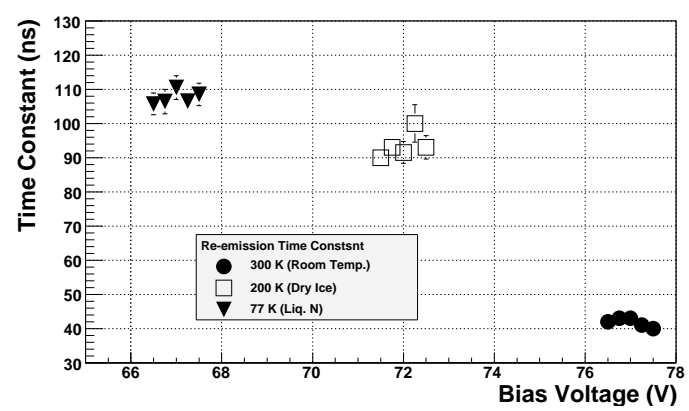

Figure 9: Re-emission time constant as a function of bias voltage. The time constant is obtained by fitting the distribution of time interval between an arbitrary pulse and the next pulse.

\section{After-pulse and Recovery}

Carrier trapped at lattice defects and delayed release after a short time causes after-pulses. We obtain the re-emission time constant by measuring the time interval between an arbitrary pulse and the next pulse $(=\mathrm{T})$. Figure 8 shows the distribution of $\mathrm{T}$ taken at $200 \mathrm{~K}$. The distribution of $\mathrm{T}$ consists of two components; the dark noise and the after-pulse. As a result of fitting by sum of two exponentials, we obtain the re-emission time constant of after-pulsing. Figure 9 shows the time constant as a function of the bias voltage. At low temperature, the time constant is found to be longer than that of room temperature. Bias voltage dependence at each temperature is also found to be small from the Figure 9 .

The last topic is the recovering process. Since after-pulsing is occurred in the same pixel where the original breakdown happens, we can probe the recovery process with the after-pulsing. Although the after-pulse piles up on the original pulse, waveform analysis is powerful for separating these two pulses [8]. Figure 10 shows the waveforms taken at $77 \mathrm{~K}$. In the case of low temperature, 


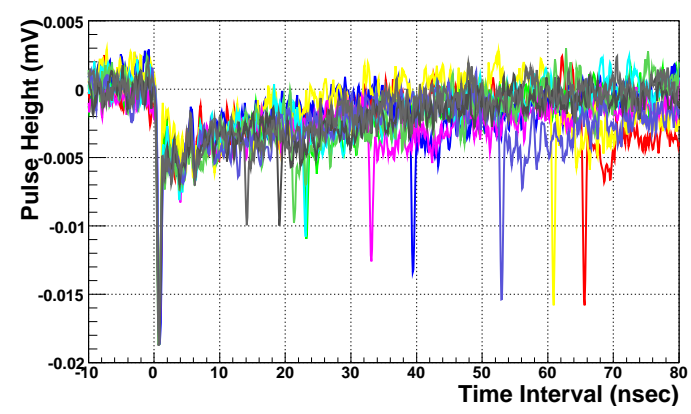

Figure 10: Waveforms taken at $77 \mathrm{~K}$. The envelope curve of the peak position of after-pulses indicates the existence of the recovering.

the recovery time is observed to be longer and the fast component is apparent, therefore the recovery process can be clearly seen.

\section{Conclusion}

In this paper, we have reported the basic properties of MPPC at low temperatures (200K and $77 \mathrm{~K})$. We observed the characteristic waveform at low temperature and measured the breakdown voltage and the rates of dark noise. We also evaluated the cross-talk, the after-pulse and the recoverying. All the measured items can be explained by knowledge obtained so far except for the fast component in the observed waveform at low temperature. We plan to study the avalanching and quenching processes more precisely.

\section{Acknowledgment}

The authors wish to express our deep appreciation to the KEK-DTP photon-sensor group members for helpful discussion and suggestion.

\section{References}

[1] D. Renker "Geiger-mode avalanche photodiodes, history, properties and problems" Nucl. Instrum. Meth. A 567 (2006) 48-56

[2] Hamamatsu Photonics K.K. http://jp.hamamatsu.com/products/sensor-ssd/4010/index_en.html

[3] P. Buzhan et al., "Large area silicon photomultipliers: Performance and applications", Nucl. Instrum. Meth. A 567 (2006) 78-82

[4] G. Bertolini and A. Coche, "Semiconductor Detectors", Elsevier-North Holland, Amsterdam, 1968.

[5] R.Newman, W.C.Dash, R.N.Hall and W.E.Bursh, "Visible light from a silicon p-n junction" Phys. Rev. Vol. 98 A,p. 1536,1955.

[6] A.L. Lacaita, F. Zappa, Bigliardi, S. Manfredi, M. " On the bremsstrahlung origin of hot-carrier-induced photons insilicon devices” IEEE Trans. Electron Devices, Vol.40, p577, 1993.

[7] Cova, S. Lacaita, A. Ripamonti, G “ Trapping phenomena in avalanche photodiodes on nanosecond scale". IEEE Trans. Electron Devices, Vol.12, p685, 1991.

[8] H. Oide et al., "Study of afterpulsing of MPPC with waveform analysis". in these Proceedings. 